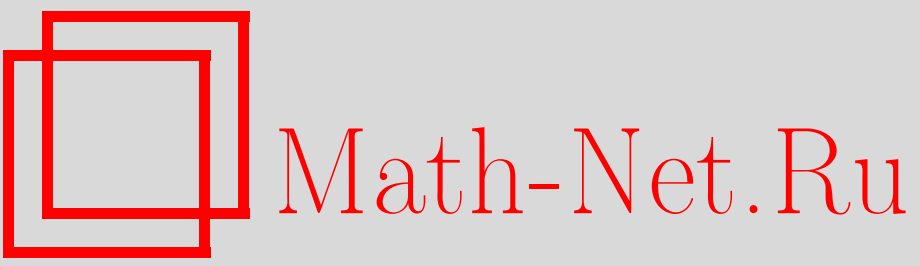

Г. Г. Амосов, В. И. Манько, Эволюция вероятностных мер, связанных с квантовыми системами, ТМФ, 2005, том 142, номер 2, 365-370

DOI: https://doi.org/10.4213/tmf1788

Использование Общероссийского математического портала Math-Net.Ru подразумевает, что вы прочитали и согласны с пользовательским соглашением

http://www . mathnet.ru/rus/agreement

Параметры загрузки:

IP: 54.196.121.252

26 апреля 2023 г., 13:45:37 


\author{
ТЕОРЕТИЧЕСКАЯ \\ И МАТЕМАТИЧЕСКАЯ \\ ФИЗИКА \\ Том 142, № 2 \\ февраль, 2005
}

(C) 2005 г.

Г.Г. Амосов*, В.И. Манько ${ }^{\dagger}$

\title{
ЭВОЛЮЦИЯ ВЕРОЯТНОСТНЫХ МЕР, СВЯЗАННЫХ С КВАНТОВЫМИ СИСТЕМАМИ
}

Выведено эволюционное уравнение для распределений вероятностей и характеристических функций квантовых томограмм, связанных с линейными и нелинейными эволюционными уравнениями для квантовых состояний.

Ключевые слова: квантовые томограммы, эволюционные уравнения.

\section{1. ВВЕДЕНИЕ}

Недавно была дана формулировка квантовой механики (вероятностное представление), в которой квантовые состояния связываются со стандартными плотностями распределений вероятностей (так называемыми томографическими плотностями, или симплектическими томограммами). Этот формализм, содержащий такую же информацию, как волновая функция и матрища плотности, основан на томографическом подходе к измерению квантовых состояний [1], [2]. В работах [1] было найдено, что томографическое распределение вероятностей связано с функцией Вигнера [3]. Функция Вигнера $W(q, p)$ дает описание квантовой механики, наиболее сходное с классической статистической механикой гамильтоновых систем. При этом выполняется условие нормировки $\int d q \wedge$ $d p W(q, p)=1$. Тем не менее $W(q, p)$ не является положительно определенной. Таким образом, она определяет квазивероятностное распределение. С другой стороны, в квантовой теории информации произвольной эрмитовой наблюдаемой может быть сопоставлено распределение вероятностей [4]. В наших недавних работах [5], [6] была установлена связь томографической плотности распределения с мерой, используемой в квантовой теории информации. Было показано, что производная такой меры определяет томографическую плотность распределения. Цель предлагаемой работы - изучить глубже свойства вероятностного представления квантовых состояний и вывести нелинейное эволюционное уравнение для квантово-вероятностных распределений, связанных с

\footnotetext{
* Московский физико-технический институт, г. Долгопрудный, Московская обл., Россия. E-mail: amosov@fizteh.ru

† Физический институт им. П. Н. Лебедева РАН, Москва, Россия. E-mail: manko@sci.lebedev.ru
} 
квантовыми состояниями, используя известное эволюционное уравнение для томографической вероятности. Заметим, что эволюционные уравнения для распределений вероятностей, которые мы выводим, принадлежат к типу, не встречавшемуся в теории классических случайных процессов. Тем самым мы не можем определить немедленно такие специфические свойства введенных нами случайных процессов, как стационарность, марковость и т.п. Тем не менее основное достоинство нашего подхода заключается в возможности использования техники классической теории вероятностей для изучения квантовых эффектов при помощи введенных нами классических случайных процессов. Для квантовой эволюции в линейном случае такое уравнение было найдено в работах [5], [6]. Для томографических плотностей распределений вероятностей в работах [7] были получены эволюционные уравнения для конденсата Бозе-Эйнштейна и для солитонов, появляюшихся в нелинейном уравнении Шредингера. В настоящей работе мы опираемся на характеристические функции, определяюшие распределение вероятностей, по аналогии с подходом, предложенным в работе [8]. Мы выводим эволюционное уравнение именно для характеристических функций, отвечающих симплектическим томограммам.

\section{2. ВЕРОЯТНОСТНЫЕ МЕРЫ, СВЯЗАННЫЕ С КВАНТОВЫМИ СИСТЕМАМИ}

Пусть $\mathcal{H}$ есть комплексное гильбертово пространство. Обозначим через $\sigma(\mathcal{H})$ множество всех состояний (операторов плотности) в $\mathcal{H}$. Элементами $\sigma(\mathcal{H})$ являются положительные операторы с единичным следом в $\mathcal{H}$. Для фиксированного $\hat{\rho} \in \sigma(\mathcal{H})$ функция трех действительных параметров $X, \mu, \nu$, определенная формулой

$$
\omega(X, \mu, \nu)=\omega_{\rho}(X, \mu, \nu)=\operatorname{Tr}(\hat{\rho} \delta(X-\mu \hat{x}-\nu \hat{p})),
$$

называется квантовой томограммой. Здесь мы использовали операторную $\delta$-функцию, определенную преобразованием Фурье как

$$
\delta(\hat{a})=\frac{1}{2 \pi} \int_{\mathbb{R}} e^{i k \hat{a}} d k .
$$

Для любых фиксированных $\mu$ и $\nu$ томограмма $\omega(X, \mu, \nu)$ представляет собой плотность распределения вероятностей, так что

$$
\omega(X, \mu, \nu) \geqslant 0, \quad \int_{\mathbb{R}} \omega(X, \mu, \nu) d X=1 .
$$

Функция распределения $\mathcal{M}(X, \mu, \nu)=\mathcal{M}_{\rho}(X, \mu, \nu)$, отвечаюшая плотности $\omega_{\rho}(X, \mu, \nu)$, может быть получена следуюшим образом. Рассмотрим спектральное разложение наблюдаемой $\mu \hat{x}+\nu \hat{p}$ :

$$
\mu \hat{x}+\nu \hat{p}=\int_{\mathbb{R}} X d \widehat{M}((-\infty, X])=\int_{\mathbb{R}} X \delta(X-\mu \hat{x}-\nu \hat{p}) d X
$$


где $\widehat{M}$ есть ортогональное разложение единицы, связанное с оператором $\mu \hat{x}+\nu \hat{p}$. Тогда формула

$$
\mathcal{M}(X, \mu, \nu)=\operatorname{Tr}(\hat{\rho} \widehat{M}((-\infty, X]))
$$

определяет функцию распределения для плотности $\omega(X, \mu, \nu)$, так что

$$
\omega(X, \mu, \nu)=\frac{d \mathcal{M}(X, \mu, \nu)}{d X}
$$

Физический смысл распределения вероятностей, определяемого функцией $\mathcal{M}(X, \mu, \nu)$ дают показания классического прибора, измеряющего наблюдаемую $\hat{x}$ (квадратуру) в системе координат фазового пространства, в которой была произведена замена масштаба и совершен поворот (квадратура гомодина). Известно, что функция распределения является инвариантом преобразования масштаба, так что $\mathcal{M}(\lambda X, \lambda \mu, \lambda \nu)=\mathcal{M}(X, \mu, \nu)$.

Пусть множество характеристических функций распределений $\omega(X, \mu, \nu)$ определяется формулой

$$
F(k, \mu, \nu)=F_{\rho}(k, \mu, \nu)=\int_{\mathbb{R}} e^{i k X} \omega_{\rho}(X, \mu, \nu) d X=\operatorname{Tr}\left(\hat{\rho} e^{i k(\mu \hat{x}+\nu \hat{p})}\right)
$$

Введенная функция обладает свойством

$$
F\left(\frac{k}{\lambda}, \lambda \mu, \lambda \nu\right)=F(k, \mu, \nu) .
$$

Свойство $\left(1^{\prime}\right)$ отвечает свойству однородности томограмм. Заметим, что можно восстановить $\omega(X, \mu, \nu)$ по $F(k, \mu, \nu)$ следуюшим образом:

$$
\omega_{\rho}(X, \mu, \nu)=\frac{1}{2 \pi} \int_{\mathbb{R}} e^{-i k X} F(k, \mu, \nu) d k .
$$

Если состояние $\rho=\rho(t)$ удовлетворяет какому-либо эволюционному уравнению, то это приводит к эволюционным уравнениям для $\omega(t, X, \mu, \nu)=\omega_{\rho(t)}(X, \mu, \nu)$, $\mathcal{M}(t, X, \mu, \nu)=\mathcal{M}_{\rho(t)}(X, \mu, \nu)$ и $F(t, k, \mu, \nu)=F_{\rho(t)}(k, \mu, \nu)$. Ранее (см. [5]) мы получили (в некоторых частных случаях) эволюционные уравнения для плотности $\omega(t, X, \mu, \nu)$ и отвечающей ей функции распределения $\mathcal{M}(t, X, \mu, \nu)$. В этой работе мы выведем эволюционные уравнения для $F(t, k, \mu, \nu)$ и $\mathcal{M}(t, X, \mu, \nu)$.

\section{3. ЭВОЛЮЦИОННОЕ УРАВНЕНИЕ \\ ДЛЯ ХАРАКТЕРИСТИЧЕСКИХ ФУНКЦИЙ В ЛИНЕЙНОМ СЛУЧАЕ}

Рассмотрим квантовую эволюцию, описываемую уравнением

$$
\partial_{t} \hat{\rho}=-i[\widehat{H}, \hat{\rho}]
$$


где $\hat{\rho} \in \sigma(H)$ и $\widehat{H}$-произвольный гамильтониан. Выведем эволюционное уравнение для характеристической функции $F(t, k, \mu, \nu)$, отвечающей эволюции состояния (2). Диф̆ференцируя по $t$, мы получаем для функции (1)

$$
\partial_{t} F(t, k, \mu, \nu)=-i \operatorname{Tr}\left([\widehat{H}, \hat{\rho}] e^{i k(\mu \hat{x}+\nu \hat{p})}\right)=i \operatorname{Tr}\left(\hat{\rho},\left[\widehat{H}, e^{i k(\mu \hat{x}+\nu \hat{p})}\right]\right) .
$$

Принимая во внимание, что $e^{i k(\mu \hat{x}+\nu \hat{p})}=e^{i k \mu \hat{x}} e^{i k \nu \hat{p}} e^{k \mu \nu / 2}=e^{i k \nu \hat{p}} e^{i k \mu \hat{x}} e^{-k \mu \nu / 2}$, находим

$$
\begin{aligned}
& \operatorname{Tr}\left(\hat{\rho} \hat{p}^{s} e^{i k(\mu \hat{x}+\nu \hat{p})}\right)=\left(e^{-k \mu \nu / 2} \frac{1}{i k} \partial_{\nu} e^{k \mu \nu / 2}\right)^{s} \operatorname{Tr}\left(\hat{\rho} e^{i k(\mu \hat{x}+\nu \hat{p})}\right), \\
& \operatorname{Tr}\left(\hat{\rho} e^{i k(\mu \hat{x}+\nu \hat{p})} \hat{p}^{s}\right)=\left(e^{k \mu \nu / 2} \frac{1}{i k} \partial_{\nu} e^{-k \mu \nu / 2}\right)^{s} \operatorname{Tr}\left(\hat{\rho} e^{i k(\mu \hat{x}+\nu \hat{p})}\right), \\
& \operatorname{Tr}\left(\hat{\rho} \hat{x}^{s} e^{i k(\mu \hat{x}+\nu \hat{p})}\right)=\left(e^{-k \mu \nu / 2} \frac{1}{i k} \partial_{\mu} e^{k \mu \nu / 2}\right)^{s} \operatorname{Tr}\left(\hat{\rho} e^{i k(\mu \hat{x}+\nu \hat{p})}\right), \\
& \operatorname{Tr}\left(\hat{\rho} e^{i k(\mu \hat{x}+\nu \hat{p})} \hat{x}^{s}\right)=\left(e^{k \mu \nu / 2} \frac{1}{i k} \partial_{\mu} e^{-k \mu \nu / 2}\right)^{s} \operatorname{Tr}\left(\hat{\rho} e^{i k(\mu \hat{x}+\nu \hat{p})}\right),
\end{aligned}
$$

$s=1,2,3, \ldots$ Следовательно, если гамильтониан имеет вид $\widehat{H}=W(\hat{p})+V(\hat{x})$, где $W$ и $V$-произвольные аналитические функции, то эволюционное уравнение для $F(t, k, \mu, \nu)$ принимает форму

$$
\begin{gathered}
\partial_{t} F(t, k, \mu, \nu)=i\left(W\left(e^{-k \mu \nu / 2} \frac{1}{i k} \partial_{\nu} e^{k \mu \nu / 2}\right)-W\left(e^{k \mu \nu / 2} \frac{1}{i k} \partial_{\nu} e^{-k \mu \nu / 2}\right)\right) F(t, k, \mu, \nu)+ \\
+i\left(V\left(e^{-k \mu \nu / 2} \frac{1}{i k} \partial_{\mu} e^{k \mu \nu / 2}\right)-V\left(e^{k \mu \nu / 2} \frac{1}{i k} \partial_{\mu} e^{-k \mu \nu / 2}\right)\right) F(t, k, \mu, \nu)
\end{gathered}
$$

В частном случае $W(\hat{p})=\hat{p}^{2} / 2$, получаем

$$
\begin{aligned}
\partial_{t} F(t, k, \mu, \nu)= & \mu \partial_{\nu} F(t, k, \mu, \nu)+i\left(V\left(e^{-k \mu \nu / 2} \frac{1}{i k} \partial_{\mu} e^{k \mu \nu / 2}\right)-\right. \\
& \left.-V\left(e^{k \mu \nu / 2} \frac{1}{i k} \partial_{\mu} e^{-k \mu \nu / 2}\right)\right) F(t, k, \mu, \nu) .
\end{aligned}
$$

\section{4. НЕЛИНЕЙНОЕ ЭВОЛЮЦИОННОЕ УРАВНЕНИЕ}

Рассмотрим в качестве примера нелинейное эволюционное уравнение для волновой функции $\psi \in \mathcal{H}$ следуюшего вида:

$$
i \psi_{t}=\frac{\hat{p}^{2}}{2} \psi+V\left(|\psi|^{2}\right) \psi
$$


Нелинейность обусловлена зависимостью потенциальной энергии от волновой функции. Имеется известный пример нелинейного уравнения Шредингера с кубической нелинейностью [9]. Введем уравнение для комплексно-сопряженной функции $\psi^{*}$ :

$$
-i \psi_{t}^{*}=\frac{\hat{p}^{2}}{2} \psi^{*}+V\left(|\psi|^{2}\right) \psi^{*} .
$$

Тогда для состояния $\hat{\rho}(t)=|\psi(t)\rangle\langle\psi(t)|$ получается эволюционное уравнение Лиувилля-фонон Неймана

$$
\hat{\rho}_{t}=-i\left[\frac{\hat{p}^{2}}{2}, \hat{\rho}\right]-i\left[\widehat{V}\left(|\psi|^{2}\right), \hat{\rho}\right],
$$

где оператор $\widehat{V}\left(|\psi|^{2}\right)$ действует по формуле $\left(\widehat{V}\left(|\psi|^{2}\right) \phi\right)(X)=V\left(|\psi(X)|^{2}\right) \phi(X), \phi \in \mathcal{H}$.

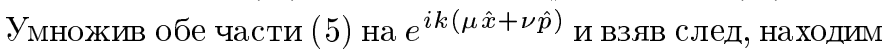

$$
\partial_{t} F(t, k, \mu, \nu)=\mu \partial_{\nu} F(t, k, \mu, \nu)+i \operatorname{Tr}\left(\hat{\rho},\left[\widehat{V}\left(|\psi|^{2}\right), e^{i k(\mu \hat{x}+\nu \hat{p}}\right]\right) .
$$

Обозначим $\widetilde{V}(X)=V\left(|\psi(X)|^{2}\right)$. Тогда из (4) следует, что

$$
\begin{aligned}
\partial_{t} F(t, k, \mu, \nu)= & \mu \partial_{\nu} F(t, k, \mu, \nu)+i\left(\widetilde{V}\left(e^{-k \mu \nu / 2} \frac{1}{i k} \partial_{\mu} e^{k \mu \nu / 2}\right)-\right. \\
& \left.-\widetilde{V}\left(e^{k \mu \nu / 2} \frac{1}{i k} \partial_{\mu} e^{-k \mu \nu / 2}\right)\right) F(t, k, \mu, \nu) .
\end{aligned}
$$

Заметим, что

$$
|\psi(X)|^{2}=\omega(X, 1,0)=\frac{1}{2 \pi} \int_{\mathbb{R}} e^{-i k X} F(t, k, 1,0) d k .
$$

Таким образом, получается эволюционное уравнение вида

$$
\begin{aligned}
& \partial_{t} F(t, k, \mu, \nu)=\mu \partial_{\nu} F(t, k, \mu, \nu)+ \\
& \quad+i\left\{V\left(\frac{1}{2 \pi} \int_{\mathbb{R}} \exp \left(-i \tilde{k} e^{-k \mu \nu / 2} \frac{1}{i k} \partial_{\mu} e^{k \mu \nu / 2}\right) F(t, \tilde{k}, 1,0) d \tilde{k}\right)-\right. \\
& \left.\quad-V\left(\frac{1}{2 \pi} \int_{\mathbb{R}} \exp \left(-i \tilde{k} e^{k \mu \nu / 2} \frac{1}{i k} \partial_{\mu} e^{-k \mu \nu / 2}\right) F(t, \tilde{k}, 1,0) d \tilde{k}\right)\right\} F(t, k, \mu, \nu) .
\end{aligned}
$$

Используя обратное преобразование Фурье, из уравнения (6) можно вывести эволюционное уравнение для распределения вероятностей $\mathcal{M}(t, X, \mu, \nu)$ :

$$
\begin{aligned}
\partial_{t} \mathcal{M}(t, X, \mu, \nu)= & \mu \partial_{\nu} \mathcal{M}(t, X, \mu, \nu)+i\left\{V \left(\frac { 1 } { 2 \pi } \int _ { \mathbb { R } } \operatorname { e x p } \left(-\frac{\partial}{\partial \tilde{X}} e^{-(i \mu \nu / 2)(\partial / \partial X)} \times\right.\right.\right. \\
& \left.\left.\times\left(\frac{\partial}{\partial X}\right)^{-1} \partial_{\mu} e^{(i \mu \nu / 2)(\partial / \partial X)}\right) d \mathcal{M}(t, \tilde{X}, 1,0)\right)- \\
& -V\left(\frac { 1 } { 2 \pi } \int _ { \mathbb { R } } \operatorname { e x p } \left(-\frac{\partial}{\partial \widetilde{X}} e^{(i \mu \nu / 2)(\partial / \partial X)} \times\right.\right. \\
& \left.\left.\left.\times\left(\frac{\partial}{\partial X}\right)^{-1} \partial_{\mu} e^{-(i \mu \nu / 2)(\partial / \partial X)}\right) d \mathcal{M}(t, \widetilde{X}, 1,0)\right)\right\} \mathcal{M}(t, X, \mu, \nu) .
\end{aligned}
$$

Здесь мы обозначили через $(\partial / \partial X)^{-1}$ оператор, определенный формулой

$$
\left\{\left(\frac{\partial}{\partial X}\right)^{-1} f\right\}(x)=\frac{1}{2 \pi} \int_{\mathbb{R}} d k \int_{\mathbb{R}} d y e^{i k(y-x)} \frac{1}{i k} f(y) .
$$

7 Теоретическая и математическая физика, т. 142, № 2, 2005 г. 


\section{5. ЗАКЛЮЧЕНИЕ}

Мы вывели эволюционные уравнения для распределений вероятностей и их характеристических функций, связанных с квантовой эволюцией, в двух случаях: для линейного эволюционного уравнения с гамильтонианом вида $\widehat{H}=W(\hat{p})+V(\hat{x})(4)$ и для эволюции, определяемой нелинейным уравнением Шредингера (6).

\section{Список литературы}

[1] J. Bertrand, P. Bertrand. Found. Phys. 1987. V. 17. P. 397; K. Vogel, H. Risken. Phys. Rev. A. 1989. V. 40. P. 2847.

[2] D. T. Smithey, M. Beck, M. G. Raymer, A. Faridani. Phys. Rev. Lett. 1993. V. 70. P. 1244.

[3] E.P. Wigner. Phys. Rev. 1932. V. 40. P. 749.

[4] A.S. Holevo. Statistical structure of quantum theory. Lect. Notes Phys. V. 67. Berlin: Springer, 2001.

[5] G. G. Amosov, V.I. Man'ko. Phys. Lett. A. 2003. V. 318. P. 287.

[6] G. G. Amosov, V. I. Man'ko. J. Russ. Laser Res. 2003. V. 25. P. 253; Quantum tomograms as densities of von Neumann probability diistributions. In: Squeezed States and Uncertainty Relations. Proc. of the 8th Int. Conf. on Squeezed States and Uncertainty Relations (Puebla, Mexico, June 9-13, 2003). Eds. H. Moya-Cessa et al. princeton: Rinton, 2003. P. 7.

[7] S. De Nicola, R. Fedele, M. A. Man'ko, V. I. Man'ko. Eur. Phys. J. B. 2003. V. 36. P. 385; J. Russ. Laser Res. 2004. V. 25. P. 1.

[8] K. E. Cahill, R. J. Glauber. Phys. Rev. 1969. V. 177. P. 1882.

[9] Л. А. Тахтаджсян, Л. Д. Фаддеев. Гамильтонов подход в теории солитонов. М.: Наука, 1986. 